\title{
The ORFEUS Far Ultraviolet Spectrum of the LMC Binary Star HDE 269546
}

\author{
H. Widmann ${ }^{1}$, G. Krämer ${ }^{1}$, I. Appenzeller ${ }^{2}$, J. Barnstedt ${ }^{1}$, A. Fromm ${ }^{1}$, \\ M. Gölz ${ }^{1}$, M. Grewing ${ }^{3}$, W. Gringel ${ }^{1}$, C. Haas ${ }^{1}$, W. Hopfensitz ${ }^{1}$, \\ N. Kappelmann ${ }^{1}$, J. Krautter ${ }^{2}$, A. Lindenberger ${ }^{1}$, and H. Mandel ${ }^{2}$ \\ 1 Institut für Astronomie und Astrophysik, D-72076 Tübingen, Germany \\ 2 Landessternwarte Heidelberg, Königsstuhl, D-69117 Heidelberg, Germany \\ 3 Institut de Radio Astronomie Millimétrique, F-38406 St. Martin d'Hères, France
}

\begin{abstract}
Far UV high resolution spectra of $3 \mathrm{LMC}$ and SMC stars were obtained with the Echelle spectrograph during the second ORFEUS mission in Dec. 1996. We present the first results from observations of the LMC star HDE 269546. We find definitely components of very hot gas identified as OVI and SVI absorption in the galactic halo of the Milky Way and in the LMC. Additionally, more than 30 ions of the most abundant elements in different stages of ionization can be identified in both our galaxy and the LMC. For the first time we can identify a significant absorption component of molecular hydrogen in the ORFEUS II Echelle spectrum with a redshift of $200 \mathrm{~km} \mathrm{~s}^{-1}$, doubtlessly to be attributed to the LMC.
\end{abstract}

\section{Introduction}

With the Copernicus satellite the first detection of OVI (Rogerson 1973) showed the presence of a widely spread hot ISM component in the Milky Way. IUE and more recently $H S T$ spectra from distant, high galactic latitude stars and from many extragalactic objects, including stars in the LMC and SMC, gave insight into the structure of the ISM in the disc and halo of our galaxy (Savage 1987, Spitzer 1990, Danly 1991) and the existence of a hot corona surrounding the Magellanic Clouds. Copernicus observations of the Lyman and Werner bands of molecular hydrogen gave a first insight into the distribution of this most abundant molecule in the ISM (Spitzer \& Jenkins 1975 , Shull \& Beckwith 1982). ORFEUS offered the first opportunity to study the very hot OVI, and the very cool $\mathrm{H}_{2}$ ISM component in greater distances and with sufficient spectral resolution for ISM studies. Results from ORFEUS I observations of the hot halo gas were published by Hurwitz et al (1995) and Hurwitz \& Bowyer (1996).

\section{Instrumentation, Observations, and Data Reduction}

The ORFEUS $1 \mathrm{~m}$-telescope is equipped with two alternatively operating spectrometers. The details about the telescope and the Echelle spectrometer 


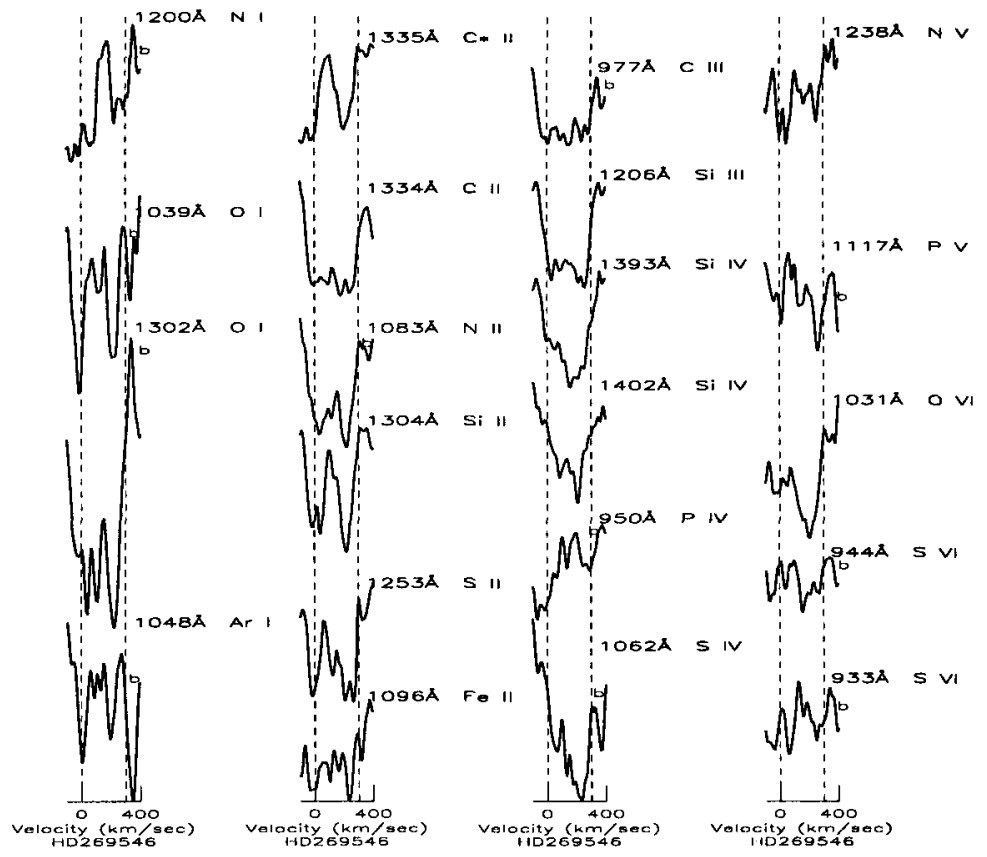

Fig. 1. A compilation of several absorption lines from neutral, singly and multiply ionized elements, including the most important O VI doublet. (b: blends)

Table 1.

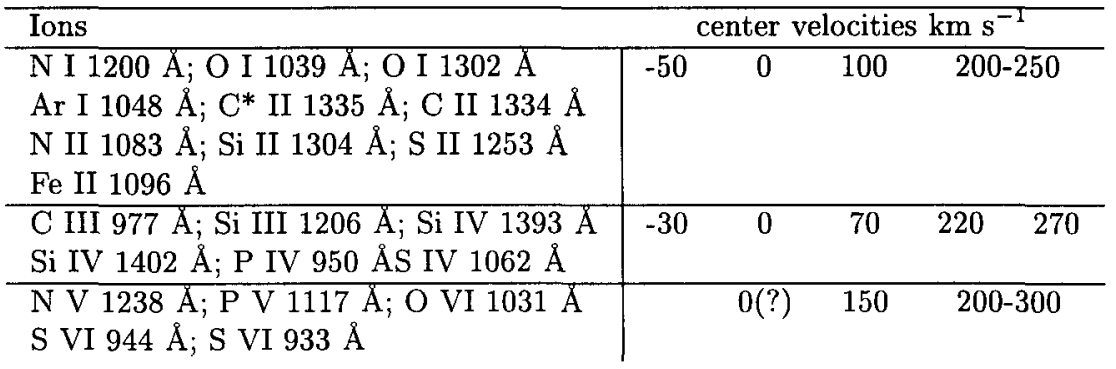

are discussed in Krämer et al (1990), the Berkeley spectrometer is described in Hurwitz \& Boywer (1995). The main properties of the Echelle spectrometer used for the measurements discussed herein are: spectral range: $912 \AA$ to $1410 \AA$; spectral resolution: $\frac{\lambda}{\Delta \lambda}>10,000$; effective area: $1 \mathrm{~cm}^{2}$. ORFEUS has been flown in orbit twice with the free flying carrier ASTRO-SPAS, launched and retrieved onboard the Shuttles STS 51, Discovery in Sept.93, and STS 80, Columbia in Nov.-Dec.96. During the ORFEUS II Mission several background stars in the LMC and SMC have been observed, three of them with 

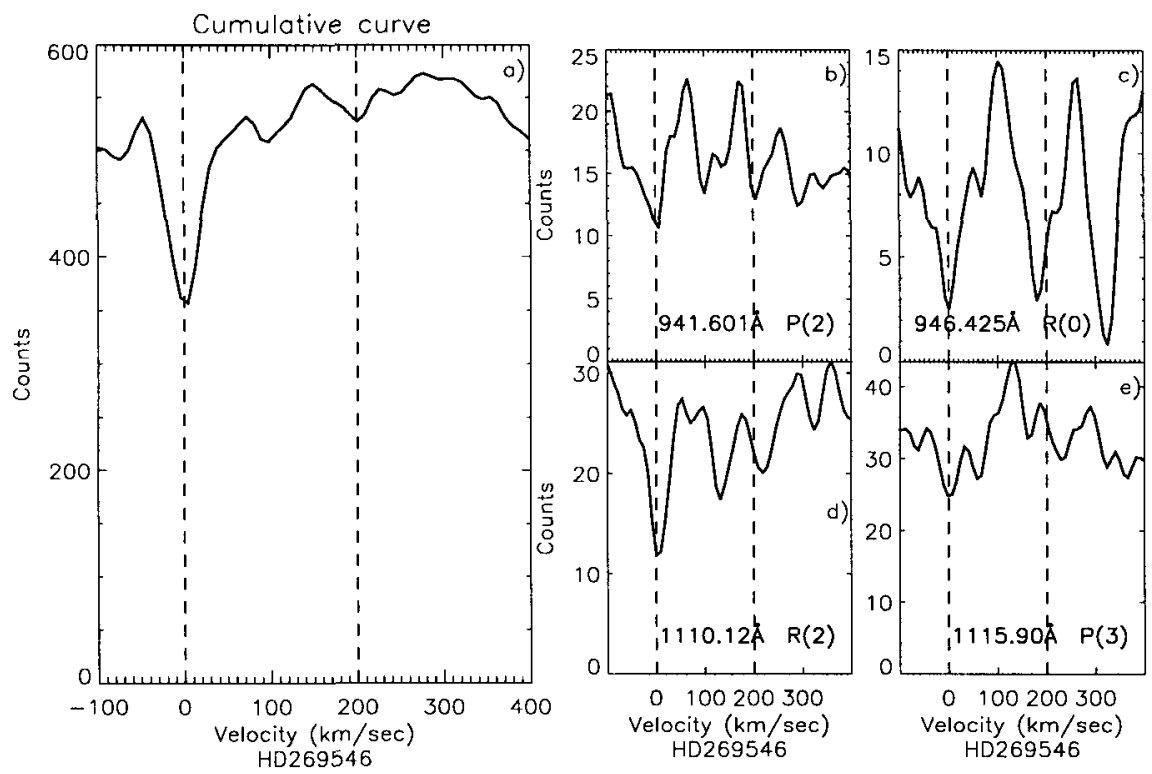

Fig. 2. a: Coaddition of 25 Lyman and Werner lines. b-e: Velocity plot of 4 different Lyman or Werner lines of molecular hydrogen.

the Echelle spectrometer. Here we present the first results from the observation of the background source HDE 269546, a B3Ia-WN3 binary that belongs to the superbubble Hen 144 (Grewing \& Schulz-Lüpertz (1980)). The total integration time of this target was $110 \mathrm{~min}$. We used a preliminary data reduction package: The intrinsic wavelengths scale is correct to about $0.1 \AA$. The velocity scale is not yet finally corrected, according to IUE spectra the scale should be shifted by about $-30 \mathrm{~km} \mathrm{~s}^{-1}$ (Chu et al (1994)). The flux scale is arbitrary.

\section{Discussion of the ion spectra}

We find in our spectrum more than 50 interstellar lines. Some of these are blended mostly with molecular hydrogen lines, but there are more than 30 lines of many important elements in different ionization stages, most of them not accessible with HST. Figure 1 shows a velocity plot of some of these lines, the spectra are smoothed with a 3 pixel boxcar, the pixel size is 0.03 $\AA$. We identify between 4 and 5 significantly different components at each ionization stage with slightly different center velocities (Tab. 1). There is also strong indication that at least at low and intermediate stages there is some material in all velocity ranges. Qualitatively we can state that the highest column densities for the neutral and weakly ionized material are found at 0 
and around $200 \mathrm{~km} \mathrm{~s}^{-1}$ to be attributed to the LMC. For the highly ionized material especially OVI and SVI the column densities are much higher at $200-250 \mathrm{~km} \mathrm{~s}^{-1}$. Summarizing the preliminary results, our measurements show a much more detailed picture about the temperature and velocity ranges of the ISM in the direction of HDE 269546 than could be derived from IUE spectra. We are working on a quantitative analysis of this spectrum and are curious about the differences and similarities in the results from the other line of sight spectra towards the LMC and SMC.

\section{Discussion of Molecular Hydrogen Lines}

In Fig. 2a-e we have compiled velocity plots of 4 different Lyman and Werner band lines of $\mathrm{H}_{2}$. In each plot we see the $\mathrm{v}=0 \mathrm{~km} \mathrm{~s}^{-1}$ component and with low significance components at about 100 and $200 \mathrm{~km} \mathrm{~s}^{-1}$. The $946 \AA$ line is blended at $200 \mathrm{~km} \mathrm{~s}^{-1}$ with another $\mathrm{H}_{2}$ line. Actually also all other lines of the rotational levels 0 and 1 are too close to each other to identify different spatial components unambigously without careful deconvolution. In order to identify significant $\mathrm{H}_{2}$ components with nonzero velocities we selected $25 \mathrm{H}_{2}$ lines from higher rotational levels which are not contaminated by any other known line within a range from -200 to $+400 \mathrm{~km} \mathrm{~s}^{-1}$ and coadded them. The result, seen in Fig. 2a, shows definitely an $\mathrm{H}_{2}$ component at about $200 \mathrm{~km}$ $\mathrm{s}^{-1}$ and, less significantly another component at about $90 \mathrm{~km} \mathrm{~s}^{-1}$. At least the higher velocity material belongs to the LMC, consistent with the neutral gas discussed in Sect. 3.

Acknowledgements. ORFEUS could only be realized with the support of all our German and American colleagues and collaborators. The ORFEUS program was supported by DARA grants WE3 OS 8501 and WE2-QV 9304and NASA grant NAG5-696.

\section{References}

Chu, Y.-H., Wakker, B., Mac Low, M.-M.Garcia-Segura, G. (1994), AJ 108, 1696 Danly, L.(1991), in IAU Symp. 144, ed H.Bloemen (Kluwer Acad. Publishers), 53 Grewing, M. et al (1980), in Proc. of the Second IUE Conference, ESA SP-157, 357 Hurwitz, M., Bowyer, S. (1995), ApJ 446, 812

Hurwitz, M., Bowyer, S. (1996), ApJ 465, 296

Hurwitz, M., Bowyer, S., Kudritzki, R.-P., Lennon, D.J. (1995), ApJ 450, 149

Krämer et al. (1990) in Observatories in Earth Orbit and Beyond, ed. Y. Kondo

(Kluwer Academic Publishers), 177

Rogerson, J.B. et al (1973), ApJ, 181, L110

Savage, B.D., Massa, D. (1987), ApJ 314, 380

Shull, J.M., Beckwith, S. (1982), ARA\&A 20, 163

Spitzer, L., Jenkins, E.B. (1975), ARA\&A 13, 133

Spitzer, L. (1990), ARA\&A 28, 71 